\title{
Factors Influencing Drug Dissolution Characteristic from Hydrophilic Polymer Matrix Tablet
}

\author{
Kiattisak Saeio', Yanee Pongpaibul', Helmut Viernstein², \\ Siriporn Okonogi ${ }^{*}$
}

\footnotetext{
${ }^{1}$ Department of Pharmaceutical Sciences, Faculty of Pharmacy, Chiang Mai University, Chiang Mai, 50200, Thailand

${ }^{2}$ Department of Pharmaceutical Technology and Biopharmaceutics, University of Vienna, A-1090, Austria
}

\begin{abstract}
The aim of this work was to investigate the factors influencing the dissolution characteristics of drug substances from hydrophilic polymer matrix tablets. Various kinds of cellulose derivative polymers were used for matrix formation. Their swelling properties were measured. The effects on drug release were studied in comparison with lactose. Results indicate that drug release characteristics from polymer matrix tablets follow Higuchi square root time kinetics. It was found that the polymers could retard the release of drugs in both simulated gastric fluid (SGF) and simulated intestinal fluid (SIF), but at different levels. The dissolution constants of drugs were higher when the $\mathrm{pH}$ of the dissolution medium was increased. Results also indicate a linear correlation between the proportions of matrix-forming structures in the tablets and the extent of dissolution retardation. It was found that not only the swelling property of the polymer but also its hydrophobicity and the hardness of the tablet played an important role in the retardation of drug release. Results also indicate that PVP K30 supports cellulose polymers so as to improve the release behavior in order to better keep drug release constant in each time interval.
\end{abstract}




\section{Keywords}

Pentoxifylline, Ketoprofen, sustained release, tablet, HPMC, PVP

\section{Introduction}

Drug modification and dosage forms have been investigated to control the time course of drug release and to maintain constant drug bioavailability. Sustained release oral dosage forms have been demonstrated to improve therapeutic efficiency by maintenance of a steady drug plasma concentration [1,2]. Moreover, there are many advantages of sustained release dosage forms such as minimization of patient compliance problems, reduction of both local and systemic side effects, avoidance of night time dosing and minimization of "peaks" and "valleys" in drug blood levels. With drugs of short half life that require frequent dosing to maintain constant therapeutic levels, the number of dose administrations can be reduced $[3,4]$.

In general, lactose is the most common excipient in tablet compression. However, "dose - dumping" may occur with soluble drugs when compressed in lactose tablets [5]. The use of polymers in controlling the release of drugs has become important in the formulation of pharmaceutical dosage forms [6,7]. One of the effective strategies to avoid the dose-dumping effect is the fabrication of sustained release tablets by incorporating the drug substance in a matrix containing a suitable rate - controlling polymer $[8,9]$. In tablet matrix systems, the tablet is in the form of a compressed compact containing an active ingredient and tablet excipients such as filler, antiadherent, and lubricant. The matrix may be tableted by direct compression of the dried powder mixture or granules $[10,11]$.

Hydrophilic polymers with high gelling capacity are of particular interest in the field of controlled release. When the tablets contact with water, polymer hydration at the solid-liquid interface occurs and is followed by formation of a viscous layer which retards the release of drug from the tablets [12,13]. However, there have been few reports on comparison among the polymer types, the dissolution media, and other factors which can influence the retardation of drug release. 
In this study, pentoxifylline (PTF) and ketoprofen (KTF) were used as model drugs. PTF is an orally active haemorrheological agent. It is indicated for improving the flow properties of blood by decreasing its viscosity [14]. It shows high solubility in water with approximately $77 \mathrm{mg} / \mathrm{ml}$ at $25^{\circ} \mathrm{C}$ and $191 \mathrm{mg} / \mathrm{ml}$ at $37^{\circ} \mathrm{C}$. The half life of the drug is only $0.8 \mathrm{~h}$ for the capsule dosage form $[15,16]$. It was reported that $98 \%$ of PTF is eliminated in $24 \mathrm{~h}$ [17]. Hence it is difficult to maintain effective drug concentrations in blood. KTF is a nonsteroidal anti-inflammatory drug with well established analgesic and antipyretic properties $[18,19]$. It is widely used for the treatment of rheumatic disorders, but requires frequent administrations to maintain therapeutic plasma concentrations [20]. In conventional prompt-release formulations, ketoprofen is rapidly and efficiently absorbed, with peak plasma levels occurring within $0.5-2 \mathrm{~h}$, after which the therapeutic plasma concentration abruptly falls to very low levels. The relatively high gastrointestinal concentration and plasma peaks associated with conventional formulations result in increased incidence of side effects [21] which demand the use of a sustained release dosage form. Various approaches have been adopted, including the use of $\mathrm{pH}$-dependent barrier films or capsules dispensed with microgranules, matrix pellets of nanocrystalline ketoprofen, and tablets. However, the effects of polymer proportion in the matrix tablet and of the $\mathrm{pH}$ of the medium on drug dissolution have not been described in detail.

The aims of the present study were to investigate the role of hydrophilic polymers in sustaining the release of drugs in tablet dosage form, and to study the effects on other drug dissolution characteristics.

\section{Experimental}

\section{Materials}

Pentoxifylline (PTF) was purchased from Laboratorio Chimico Internazionale, Italy. Hydroxyethyl cellulose (HEC) WP40 (viscosity range of 110-150 mPas), and HEC QP52000 (viscosity range of 1,800-2,100 mPas) were from Aldrich Chemical Company, Inc., USA. Hydroxypropyl methylcellulose (HPMC) E4M and HPMC F4M, 
both with viscosity range of 3,500-5,600 mPas, were supplied from Colorcon, USA. Polyvinylpyrrolidone (PVP) K25 (MW of 30,000) and PVP K30 (MW of 50,000) were from Fluka Chemika, Switzerland. Metholose $60 \mathrm{SH} 4000$ (HPMC with MW of 4,000 ) was from Shin Etsu, Japan. Anhydrous lactose was from Meggle, Germany. Ketoprofen (KTF) and other ingredients were of the highest grades available.

\section{Swelling property of the polymer}

The swelling property of cellulose derivative polymers was studied by measuring the change in volume. Certain amounts of each polymer were placed in 10-ml graduated cylinders at an initial apparent volume of 0.3-0.4 ml. Subsequently, water was added to a volume of $10 \mathrm{ml}$. The swelling property of the polymer observed within $24 \mathrm{~h}$ was expressed both as the swelling ratio and swelling rate. The swelling ratio $\left(Q_{d}\right)$ for each polymer was calculated from the following equation

$$
Q_{d}=\frac{V_{s}-V_{d}}{V_{d}}
$$

where $V_{d}$ and $V_{s}$ represent the volume of the initial dried polymer and the final volume of swollen polymer, respectively. The swelling rate was calculated as the volume increase $\left(V_{s}-V_{d}\right)$ per $\mathrm{h}$. The experiment was done in triplicate.

\section{Preparation of tablets}

Powder mixtures of PTF and polymer or lactose at a weight ratio of 2:1 were moistened and sieved through a $1.7-\mathrm{mm}$ wire mesh. After drying $\left(55^{\circ} \mathrm{C}, 8 \mathrm{~h}\right)$, the granules were sieved again through a $0.85-\mathrm{mm}$ wire mesh, then mixed with $1 \%$ talcum and $0.5 \%$ magnesium stearate. The mixture was compressed by use of a single punch tableting machine to yield tablets of $600 \mathrm{mg}$ each. The hardness of PTF tablets obtained was measured by use of a hardness tester (Pharma Test ${ }^{\circ}$, type PTB311, Germany). For the formation of KTF matrix tablets, 2 parts of KTF and 1 part of lactose were mixed with graded proportions of mixtures composed of HPMC (Metholose $60 \mathrm{SH} \mathrm{4000)}$ ) and calcium hydrogen phosphate dihydrate (mass 
ratio, 3:1). The wet mixture was granulated and sieved through a $2.0-\mathrm{mm}$ wire mesh. After drying $\left(50^{\circ} \mathrm{C}, 2 \mathrm{~h}\right)$, the granules were sieved again through a $1.0-\mathrm{mm}$ wire mesh, then mixed with $2 \%$ of $1: 1$ mixture of talcum and magnesium stearate. The mixture was compressed into double convex 11-mm diameter tablets at a pressure of $10 \mathrm{Kbar}$ using a Hanseaten E1 Excenter press. The content of KTF in each tablet was $200 \mathrm{mg}$.

\section{Dissolution studies}

Dissolution studies were performed according to Method 2 of USP at $37 \pm 0.5{ }^{\circ} \mathrm{C}$. In case of PTF, $900 \mathrm{ml}$ of simulated gastric fluid (SGF) or simulated intestinal fluid (SIF) was filled into a glass dissolution vessel and the paddle was rotated at the speed of $50 \mathrm{rpm}$. The tablet was put in the sinker (Fig. 1) prior to contact with the medium to prevent tablet floating. Determination of drug release was done by spectrophotometry at $273 \mathrm{~nm}$. In case of KTF, 1,000 ml of dissolution medium $(0.1 \mathrm{M} \mathrm{HCl}$, or buffer $\mathrm{pH} 5.5$, or buffer $\mathrm{pH} 6.8)$ was used at a paddle speed of $150 \mathrm{rpm}$. Buffer pH 5.5 comprised $56.9 \mathrm{ml} 0.2 \mathrm{M} \mathrm{Na}_{2} \mathrm{HPO}_{4}$ and $43.1 \mathrm{ml} 0.1 \mathrm{M}$ citric acid solution. Buffer $\mathrm{pH} 6.8$ was formed by neutralization of $0.1 \mathrm{M} \mathrm{HCl}$ as follows. A mixture was prepared of $97.2 \mathrm{ml} 0.1 \mathrm{M} \mathrm{HCl}+0.68 \mathrm{M} \mathrm{Na} 2 \mathrm{HPO} 4+5.25 \mathrm{M}$ $\mathrm{NaOH}$ as required. Quantification of liberated drug was performed by photometric measurement at a wavelength of $300 \mathrm{~nm}$.

(a)

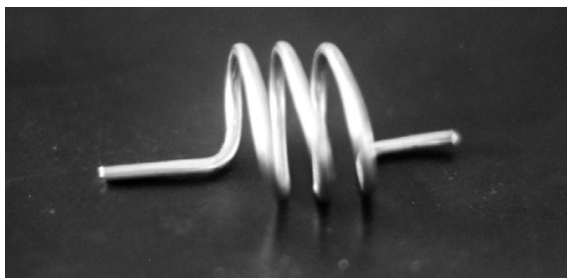

(b)

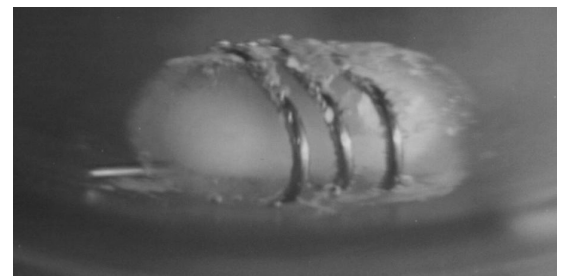

Fig. 1. Tablet sinker (a) and the sinker with a PTF tablet during dissolution (b) 


\section{Effect of PVP as a drug release modifier}

This experiment was performed with the PTF-cellulose polymer tablet. The amount of $30 \%$ polymer of the selected formulation was replaced with PVP K25 or PVP K30. Tablet preparation and dissolution study were performed as described above.

\section{Results and Discussion}

\section{Swelling properties of polymers}

The swelling of the hydrophilic polymers is associated with molecular hydration and always occurs when polymers come in contact with water. Progressive swelling of polymers causes considerable structural changes. These include the modification of porosity and tortuosity of the polymers which affect the diffusional release of a drug $[22,23]$. The swelling property of polymers can be expressed as swelling ratio or rate of swelling. The swelling ratio suggests the ability of a polymer to expand its volume after hydration whereas the rate of swelling indicates how fast the water can penetrate into the polymer crystal lattice. In the present experiments, four cellulose derivative polymers exhibited different swelling levels as shown in Tab. 1. It was demonstrated that HEC QP52000 exhibits the most swelling with the highest swelling ratio and rate of swelling of 11.66 and $0.53 \mathrm{ml} / \mathrm{h}$ respectively, followed by the insignificantly lesser level of swelling of HEC WP40 and HPMC E4M. The poorest swelling property was found with HPMC F4M.

\begin{tabular}{lcc}
\hline \multicolumn{1}{c}{ Polymers } & Swelling Ratio & Rate of Swelling $(\mathbf{m l} / \mathbf{h})$ \\
\hline HEC WP40 & $2.25 \pm 0.13$ & 0.10 \\
HEC QP52000 & $11.66 \pm 0.33$ & 0.53 \\
HPMC E4M & $2.67 \pm 0.13$ & 0.08 \\
HPMC F4M & $1.05 \pm 0.10$ & 0.04 \\
\hline
\end{tabular}

Tab. 1. Swelling ratio of all polymers within $24 \mathrm{~h}$ 


\section{Physical appearance and hardness of PTF tablets}

The physical appearance of PTF tablets prepared with different types of polymers or lactose were oblong shape and white in color. It was found that PTF matrix tablets prepared with the test polymers or lactose could be easily compressed into tablets. The tablets were easily removable from the die cavity regardless of the addition of a small quantity of talcum and magnesium stearate. The tablets compressed with HEC showed rather rough surface, while those with HPMC or lactose possessed smooth and glitter surfaces as shown in Fig. 2.

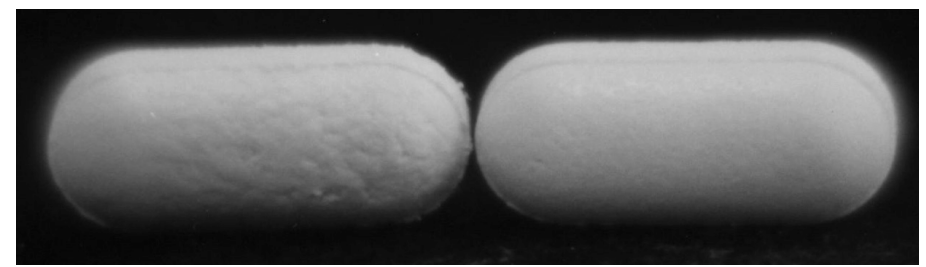

Fig. 2. The surface characteristics of a HEC tablet (left) and a HPMC tablet (right)

The tablets obtained from polymer matrix exhibited different hardness values in the range of 45-70 $\mathrm{N}$ as shown in Tab. 2, while those from lactose also fell within this range. In comparison among those excipients, HPMC E4M gave the tablets with the highest hardness. The hardness always influences the compaction of substances inside the tablets; the higher the hardness, the higher the compaction of the tablets. The high compaction causes a decrease in porosity of the polymer matrix. Hence, the tablets with high compaction have a high ability to retard the water penetration into their core. The highest hardness of HPMC E4M containing tablets indicated that they should have the highest ability to retard water penetration, resulting in slow release of drug molecules from this matrix. 


\begin{tabular}{lc}
\hline \multicolumn{1}{c}{ Excipients } & Average Hardness $^{*}(\mathrm{~N})$ \\
\hline HEC WP40 & $65.7 \pm 2.2$ \\
HEC QP52000 & $44.7 \pm 4.3$ \\
HPMC E4M & $69.8 \pm 4.2$ \\
HPMC F4M & $46.9 \pm 2.7$ \\
Lactose & $56.1 \pm 4.7$ \\
\hline & values represent average \pm SD $(\mathrm{n}=3)$
\end{tabular}

Tab. 2. Hardness of PTF tablets from different excipients

\section{Dissolution of drugs from cellulose polymer tablets}

The dissolution behavior of PTF from tablets containing different types of cellulose polymers is illustrated in Fig. 3. Results revealed a significant difference in drug dissolution behavior between tablets with lactose and those with the polymers. A very rapid release of drug from lactose tablets was found (within $30 \mathrm{~min}$ ) in both SGF and SIF whereas the polymer tablets showed the retardation of drug release. This was due to the high water solubility of lactose and the relatively low compaction of such tablets, according to its inferior hardness. As a consequence, water easily penetrates into the tablets and the soluble PTF molecules are rapidly released. The polymers are less water soluble than lactose. Hence, the water penetration into the polymer matrix tablet is more difficult. This leads to the retardation of drug release. Results also indicate that in SGF, the amount of drug released is less than in SIF whose $\mathrm{pH}$ is higher. The effect of $\mathrm{pH}$ was consistent when KTF was used as a model drug and HPMC was used as a model polymer as shown in Fig 4. The higher the $\mathrm{pH}$, the higher the drug dissolution constant $(\mathrm{y}=$ $0.123-1.73 \cdot 10^{-3} x, y=0.621-1.75 \cdot 10^{-2} x$, and $y=0.816-2.50 \cdot 10^{-2} x$ in $0.1 \mathrm{M}$ $\mathrm{HCl}$, buffer $\mathrm{pH} 5.5$, and buffer $\mathrm{pH} 6.8$, respectively). In addition, there is a linear correlation between the proportion of polymer in the tablets and the extension of drug retardation in all three dissolution media $\left(r^{2}=0.826,0.994\right.$, and 0.998 in $0.1 \mathrm{M}$ $\mathrm{HCl}$, buffer $\mathrm{pH} 5.5$, and buffer $\mathrm{pH} 6.8$, respectively). 


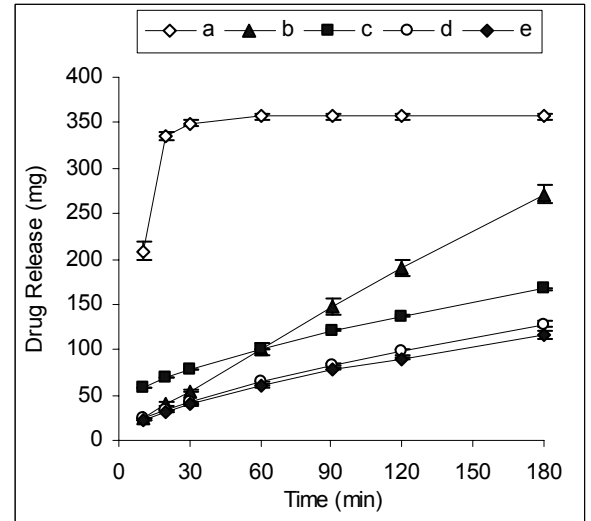

(A)

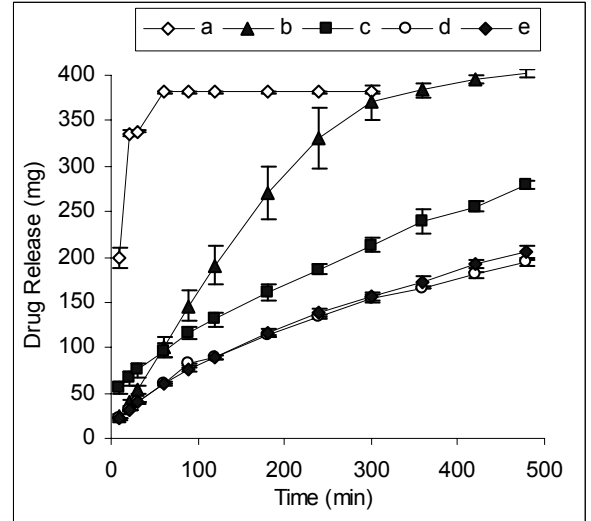

(B)

Fig. 3. Dissolution profiles of PTF release from tablets of different excipients; lactose (a), HEC WP40 (b), HEC QP52000 (c), HPMC E4M (d), and HPMC E4M (e) in SGF (A) and SIF (B)

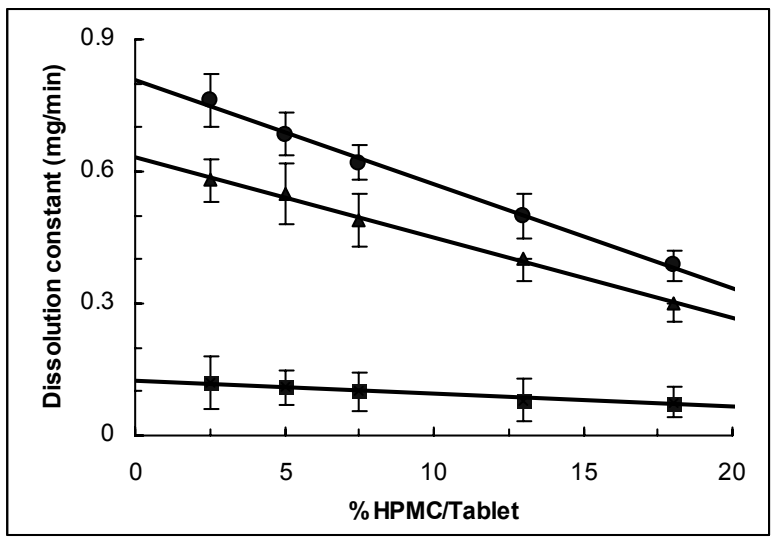

Fig. 4. The relationship of polymer content and dissolution constant of KTF in buffer pH $6.8(\bullet)$, buffer $\mathrm{pH} 5.5(\mathbf{\bullet})$, and $0.1 \mathrm{M} \mathrm{HCl}(\bullet)$ 
However, the total amount of drug released from polymer tablets when plotted versus time did not fit the regression line. This was more obviously seen when dissolution took place in SIF. Their release behavior seemed to follow the Higuchi equation as follows

$$
W=k t^{\frac{1}{2}}
$$

where $W$ is the total amount of drug release at a time $t$ and $k$ is the release constant. The Higuchi plots of all drug polymer tablets in SGF and SIF are shown in Fig. 5 and Fig. 6 respectively.

(a)

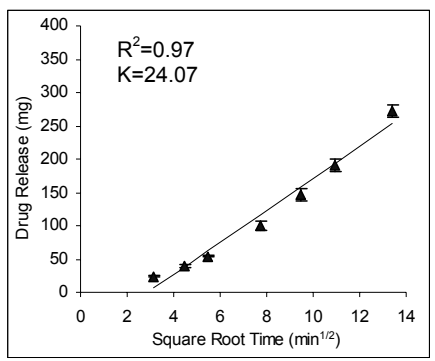

(c)

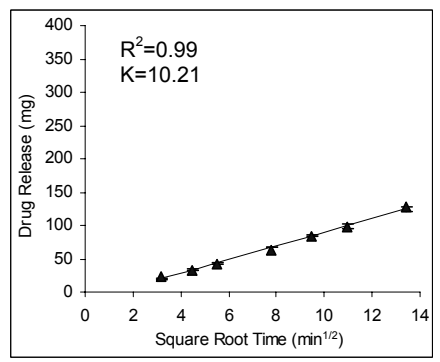

(b)

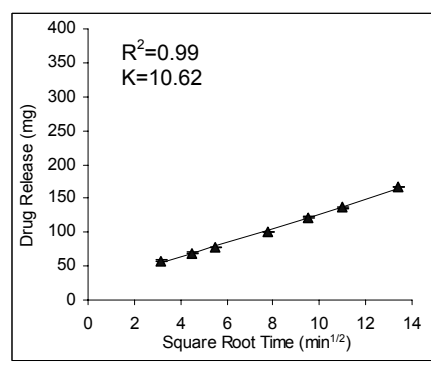

(d)

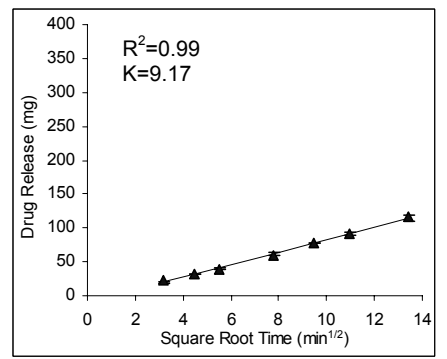

Fig. 5. Higuchi plots of PTF release from tablets containing HEC WP40 (a), HEC QP52000 (b), HPMC E4M (c) and HPMC F4M (d) in SGF

The dissolution rate of PTF from each polymer tablet could be determined from the slope of the Higuchi plot. It was obviously seen, particularly in SIF, that the drug release was better sustained in the tablets with HPMC than HEC. This may be due to the water solubility of the polymers. Based on the chemical structure, HEC 
polymer should be more water soluble than HPMC. The water could thus penetrate more easily to dissolve PTF in the HEC tablets. Moreover, the viscosity of a polymer gel is a rate-controlling factor in drug dissolution [24]. The higher the viscosity, the more resistant the matrix to dissolution and erosion. Since the HPMC polymers used in this study are much more viscous than HEC, this could explain the release retardation property of the HPMC polymers. However, it was noticed that some retardation of drug release occurred in HEC QP52000 tablets. This may be due to the high swelling property of this polymer. Once the tablets contact the medium, the penetrated water causes this polymer to swell rapidly. The thickness and decreased porosity of the diffusion layer inhibits the passage of the drug molecules, resulting in retardation of drug release.

(a)

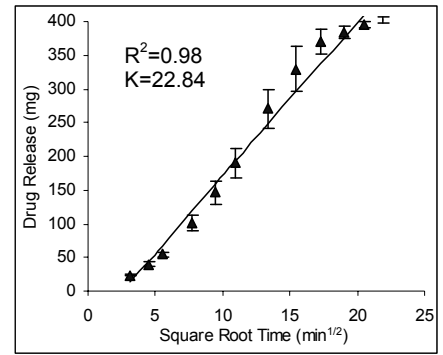

(c)

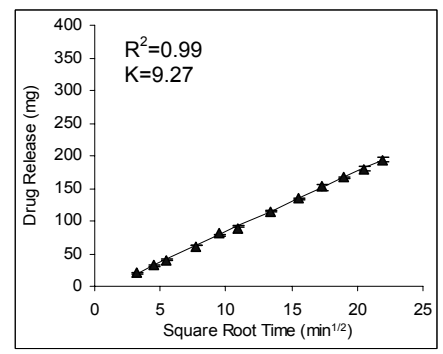

(b)
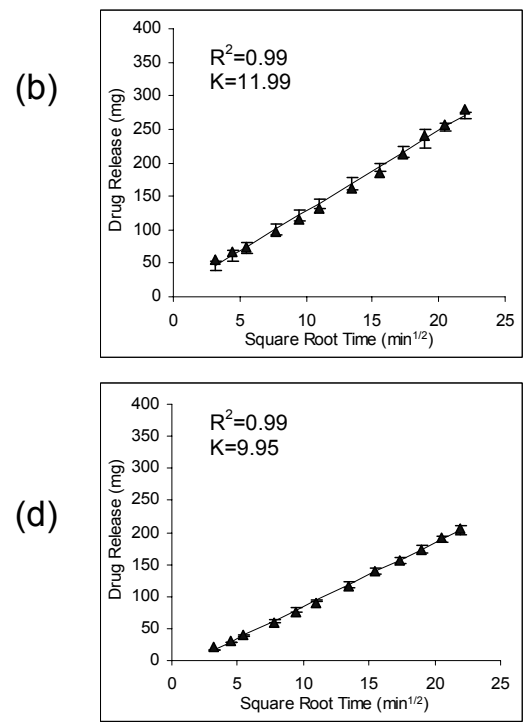

Fig. 6. Higuchi plots of PTF release from tablets containing HEC WP40 (a), HEC QP52000 (b), HPMC E4M (c) and HPMC F4M (d) in SIF 
Comparing HPMC E4M with HPMC F4M, there was no significant difference in sustained release of drug. However, tablets with HPMC E4M exhibited significantly higher hardness accompanied by high compaction. As described previously, the higher the compaction, the higher the retardation of drug release. Therefore, the drug retardation seemed to be assured when drugs were incorporated in HPMC E4M tablets. Moreover, the higher hardness provided tablets with better protection power from fragility due to weight or stress forces. Thus, hardness is one of the requirements for tablets that should be met for them to withstand handling in the logistic chain from producer to patient. For these reasons, HPMC E4M was selected to be the most suitable excipient for drug tablet formulation.

\section{Effect of PVP as drug release modifier}

Initial results indicated that HPMC E4M was the most suitable polymer base for drug matrix tablets. However, the amount of drug release in each time interval $(\mathrm{dQ} / \mathrm{dt})$ when plotted versus time $(\mathrm{t})$ was fluctuated considerably as shown in Fig. 7(a). This inconstant drug release suggested a trend of unsteady drug concentration in plasma after oral administration of this type of tablet.

(a)

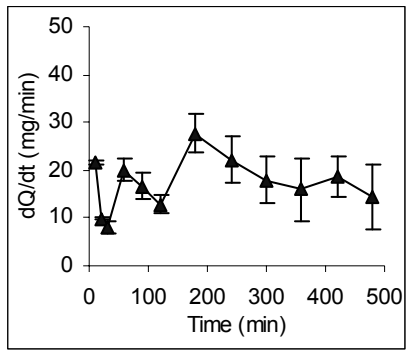

(b)

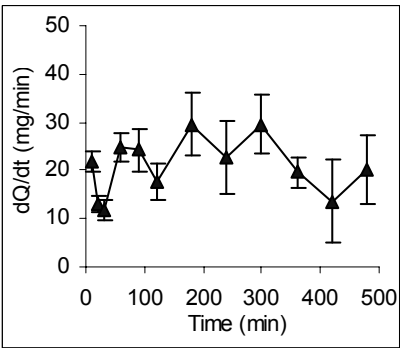

(c)

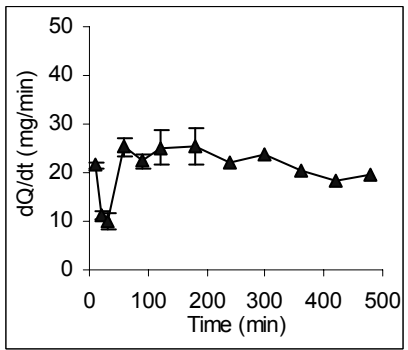

Fig. 7. Dissolution profile of drug release in each time interval $(d Q / d t)$ of PTF-HPMC E4M tablets without PVP (a), with PVP K25 (b), and with PVP K30 (c) 
As mentioned above, the release of drug from the tested polymer matrix was influenced by the dual effects of the hydrophilicity of polymer and the swelling of the matrix upon hydration. The hydrophilicity of the polymer caused the matrix to be eroded during drug dissolution. This uncontrolled erosion was considered to be a major factor for drug dissolution. If gradual constant erosion occurred, the steady drug concentration in each time interval was observed. Therefore, the fluctuated drug released was due to the uncontrolled composition of the polymer matrix.

The replacement of 30\% HPMC E4M with PVP K25 in the tablet formulation showed no effect on this release mechanism. However, when PVP K30 was used instead of PVP K25, there was some significant modification as shown in Fig. 7(c). Indeed, it was observed that the drug release profile, particularly after $60 \mathrm{~min}$ of dissolution, was more constant with the drug release at about $20 \mathrm{mg}$ during the dissolution period of $480 \mathrm{~min}$. These results suggest that PVP K30 is an efficient release modifier, improving the release behavior of PTF tablets toward more constant drug release during a sufficient length of time.

\section{Conclusion}

It was concluded that the best sustained release tablet could be produced using HPMC as hydrophilic controlling polymer. The Higuchi model could be used for prediction of drug release. The $\mathrm{pH}$ of dissolution media and the proportion of polymer in the tablet affected the dissolution characteristics of the drug. It was found that not only the swelling property of the polymer but also its hydrophobicity and the hardness of the tablet played an important role in retardation of drug release. The addition of PVP K30 to the HPMC tablets improved the HPMC matrix structure so as to release the drug in constant amounts in each time interval.

\section{Acknowledgement}

Appreciation is expressed to Siam Pharmaceutical Co. Ltd. (Thailand) for supply of chemicals and partial financial support. 


\section{References}

[1] Turner S, Federici C, Hite M, Fassihi R.

Formulation Development and Human In Vitro-In Vivo Correlation for a Novel, Monolithic Controlled - Release Matrix System of High Load and Highly WaterSoluble Drug Niacin.

Drug Dev. Ind. Pharm. 2004;30(8):797-807.

[2] Fernandes CM, Ramos P, Amilcar CF, Veiga FB.

Hydrophilic and hydrophobic cyclodextrins in a new sustained release oral formulation of nicardipine: in vitro evaluation and bioavailability studies in rabbits.

J. Control. Release 2003;88(1):127-134.

[3] Greenblatt DJ.

Pharmacokinetic determinants of hypnotic drug action: The art and science of controlling release.

Sleep Medicine 2006;7S1:S10-14.

[4] Pather SI, Russell I, Syce JA, Neau SH.

Sustained release theophylline tablets by direct compression: Part 1 :

formulation and in vitro testing.

Int. J. Pharm. 1998;164(1):1-10.

[5] Sako K, Sawada T, Nakashima H, Yokohama S, Sonobe T.

Influence of water soluble fillers in hydroxypropylmethylcellulose matrices on in vitro and in vivo drug release.

J. Control. Release 2002;81(1-2):165-172.

[6] Chowdary KP, Sankar GG.

Eudragit microcapsules of nifedipine and its dispersions in HPMC-MCC: physicochemical characterization and drug release studies.

Drug Dev. Ind. Pharm. 1997;23(3):325-330. 
[7] Ebube NK, Hikal AH, Wyandt CM, Beer DC, Jones AB.

Effect of Drug, Formulation, and Process Variables on Granulation and Compaction Characteristics of Heterogeneous Matrices. Part 2. HPMC and PVP Systems.

Drug Dev. Ind. Pharm. 1996;22(7):561-567.

[8] Lotfipour F, Nokhodchi A, Saeedi M, Norouzi-Sani S, Sharbafi J, Siahi-Shadbad MR.

The effect of hydrophilic and lipophilic polymers and fillers on the release rate of atenolol from HPMC matrices.

IL Farmaco. 2004;59:819-825.

[9] Vazquez MJ, Perez-Marcos B, Gomez-Amoza JL, Martinez-Pacheco R, Souto C, Concheiro A.

Influence of Technological Variables on Release of Drug from Hydrophilic Matrices.

Drug Dev. Ind. Pharm. 1991;18(11\&12):1355-1375.

[10] Harcum WW, Skinner GW, Altekar M, Joneja SK, Barnum PE, Guo JH. Modelling the Effects of Hydroxypropylcellulose in Acetaminophen Tablet Formulation.

Drug Dev. Ind. Pharm 1998;24(10):911-918.

[11] Pabon CV, Frutos P, Lastres JL, Frutos G.

In vitro study of mixed controlled release matrix tablets containing HMPC and polyamide 12.

Drug Dev. Ind. Pharm. 1992;18(20):2163-2171.

[12] Chirico S, Dalmoro A, Lamberti G, Russo G, Titomanlio G. Analysis and modeling of swelling and erosion behavior for pure HPMC tablet. J. Pharm. Sci. 2007;122:181-188. 
[13] Vazquez MJ, Casalderrey M, Gomez-Amoza JG, Martinez-Pacheco R. Souto ,C. and Concheiro A.

Atenolol release from hydrophilic matrix tablets with hydroxypropylmethylcellulose (HPMC) mixtures as gelling agent: effects of the viscosity of HTML mixture.

Eur. J. Pharm. Sci. 1996;4:39-48.

[14] Alan W, Stephen PC.

Pentoxifylline - A Review of its Pharmacodynamic and Pharmacokinetic

Properties and its Therapeutic Efficacy.

Drug 1987;34:50- 97.

[15] Makoto O, Yoshihida M.

Controlled Drug Release of Highly Water Soluble Pentoxifylline from Time Limit Disintegration Type Wax Matrix Tablet.

Pharm. Res. 1994;11:351-354.

[16] Makoto O, Yoshihida M.

Programable Drug Release of Highly Water-Soluble Pentoxifylline from Dry Coated Wax Matrix.

J. Pharm. Sci. 1995;84(4):443-446.

[17] Gleixner K, Muller R, Lehrach F.

Pharmaceutical compositions.

U.S. Patent 4,189469 Feb. 19. 1980

[18] Fossgreen J, Kirchheiner B, Petersen FO, Tophøj E, Zachariae E.

Clinical evalutation of ketoprofen (19.583 R.P.)

In: rheumatoid arthritis-double-blind cross-over comparisonwith indomethacin.

Scand. J. Rheumatol. Suppl. 1976;14:93-98.

[19] Cathcart BJ, Vince JD, Gordon AJ, Bell M, Chalmers IM.

Studies on 2-(3-benzoylphenyl) propionic acid ('Orudis').

Ann. Rheum. Dis. 1973;32:62-65. 
[20] Liversidge GG.

Ketoprofen.

In: Florey, K. (Ed.), Analytical Profiles of Drug Substances.

New York:Accademic Press, 1981:443-471.

[21] Graham GG, Day RO, Champion GD, Lee E, Newton K.

Aspects of the clinical pharmacology of nonsteroidal anti-inflammatory drugs.

Clin. Rheum. Dis. 1984;10:229-249.

[22] Korsmeyer RW, Gurny R, Doelker E, Buri P, Peppas NA.

Mechanisms of solute release from porous hydrophilic polymers.

Int. J. Pharm. 1983;15:25-35.

[23] Korsmeyer RW, Peppas NA.

Effect of the morphology of hydrophilic polymeric matrices on the diffusion and release of water soluble drugs.

J. Membrane Sci. 1981;9(3):211-227.

[24] Emeje MO, Kunle OO, Ofoefule SI.

The effect of the molecular size of carboxymethylcellulose on the rate of hydration, matrix erosion and drug release.

Drug Delivery Tech. 2005;5:56-60. 